\title{
Measurement of Room Impulse Response Using Image Source Method
}

\author{
Prarthan Mehta ${ }^{1}$ and Vaishalee Bhadradiya ${ }^{2}$ \\ ${ }^{1}$ Dept. of Ele. \& Comm. Eng., Faculty of Technology, Dharmsinh Desai University, Nadiad-387001. India \\ ${ }^{2} \mathrm{R} \&$ D, Faculty of Technology, Dharmsinh Desai University, Nadiad-387001. India
}

\begin{abstract}
There are various computer modeling techniques based on the behavior of sound as a ray or wave to measure Room Impulse Response (RIR). The Image Source Method (ISM) is used to measure the RIR for various positions of source at different frequencies. The other methods are too complex, time consuming and less accurate. Acoustic parameters such as clarity and definition are obtained from accurately measured RIR using geometric modeling method, ISM. The calculated acoustic parameters are useful to predict the characteristics of a room, to compare the effect of different absorbing materials and also for the construction of theater, opera house and church.
\end{abstract}

Keywords-image source method; room impulse response; clarity; definition

\section{INTRODUCTION}

The RIR modeling has been a subject of interest to acousticians, musicians and architects. Since the RIR describes exclusively the behavior of a room, Linear Time Invariant acoustical system at the particular location of the source and the receiver in the particular environment of a room. The RIR is transfer function between a source and a receiver which are placed in a room. From the measured RIR, different acoustical parameters such as reverberation time, clarity, definition, center time strength and many others can be derived. These parameters describe various acoustic properties of a room.

There are many methods for measurement of RIR such as boundary element method, finite element method, finite time domain difference, ray tracing, hybrid method image source method and etc. These methods are classified into two main groups: ray based method and wave based method. ISM is ray based method and so that the sound signal is considered as a sound ray. If a sound ray strikes a solid surface, it will reflect from it. This process takes place according to the reflection law of optics. The ISM can model possible reflected paths up to a certain higher order of reflection. Thus the results are more accurate for higher of reflection. The main advantage of this method is less computational complexity and easy to implement.

\section{IMAGE SOURCE MethoD}

In geometrical room acoustics, the concept of a sound wave is replaced by the concept of a sound ray. This method is based on the principle that a specular reflection can be constructed geometrically by mirroring the source in the plane of the reflecting surface. It is used to construct the mirror image of a source.

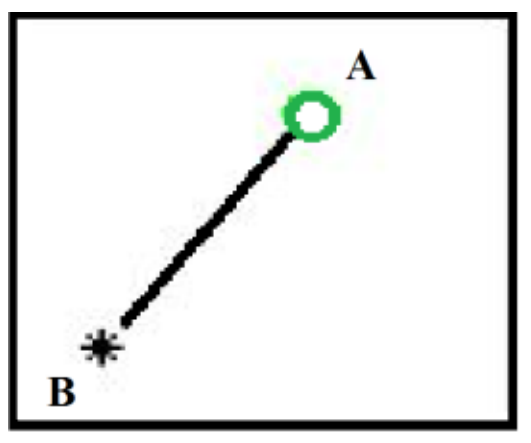

FIGURE I. TOP VIEW OF A ROOM WITH SOURCE, A AND RECEIVER, B

The figure I shows top view of a room with source and receiver. There is a point source, shown as a green circle emits the sound signal in all direction. The black star represents the location of receiver which receives the direct sound signal and each reflected sound signal from the surfaces of room. The line between source and receiver shows the direct sound signal which is received without any reflection by receiver. A mirror image of the room will be made and placed it adjacent to the original room as shown in figure II for finding possible reflected path. The image source $A^{\prime}$ which is located behind the wall, on the line perpendicular to the wall, and at the same distance from it as the original source A. Here the wall between $A \& A^{\prime}$ acts as mirror and $A^{\prime}$ is mirror image of original source A. So this mirror image of A will be referred as a virtual source, shown as black circle in figure II.

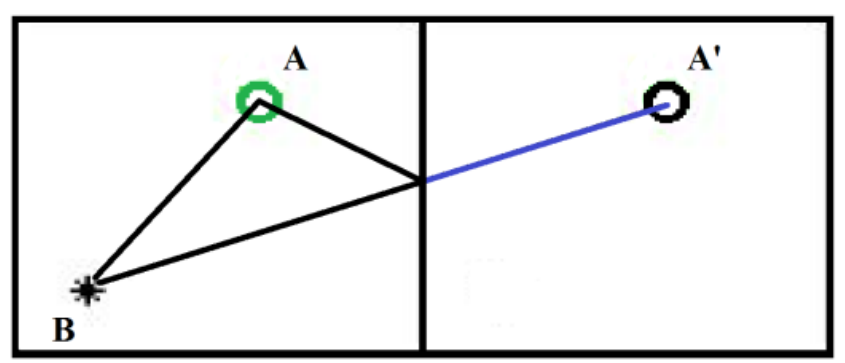

FIGURE II. TOP VIEW OF ORIGINAL ROOM AND ITS FIRST ORDER IMAGE ROOM

Assume that the image source $A^{\prime}$ emits exactly the same sound signal as the original source, so that directional characteristics are symmetrical to original source A. The energy of incident and reflected sound signal are not same, because few part of energy of the incident sound signal is 
absorbed by the wall. The fraction of energy of sound energy which is not reflected is characterized by the absorption coefficient $\alpha$ of the wall. It is the ratio of the non-reflected to the incident intensity of sound signal. It depends on the material of surface and frequency of sound signal.

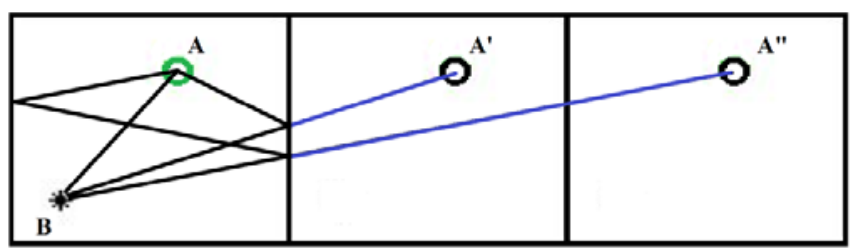

FIGURE III. TOP VIEW OF ORIGINAL ROOM WITH FIRST AND SECOND ORDER IMAGE ROOM

In the figure II, the blue line represents the perceived path of the sound signal. The higher order reflection of sound signal can be found out by repeating the mirroring process, as shown in figure III. Similarly a second order image A " is constructed which is the mirror image of $\mathrm{A}^{\prime}$ and for that the common wall acts as mirror. For this type of room shape, certain image sources are in the same order and so that it generates regular pattern of image rooms and image sources.

The path length of direct signal is distance between original source and receiver and it can be calculated from the known location of original source and receiver. The path length of reflected signal is derived from the position of image source and receiver. The position of image source is found out by using ISM. $x_{i}, y_{j}$ and $z_{k}$ are the distance between receiver and $i^{\text {th }}, j^{\text {th }}$ and $k^{\text {th }}$ position of image source in $\mathrm{x}, \mathrm{y}$ and $\mathrm{z}$ direction respectively. The $d_{i j k}$, distance is measured from Pythagorean Theorem.

$$
\begin{gathered}
d_{i j k}=\sqrt{x_{i}^{2}+y_{j}^{2}+z_{k}^{2}} \\
\mathrm{u}_{\mathrm{ijk}}=\mathrm{t}-\frac{\mathrm{d}_{\mathrm{ijk}}}{\mathrm{c}}
\end{gathered}
$$

$\mathrm{t}$ is time and $\mathrm{c}$ is the speed of sound.

$$
a_{i j k}\left(u_{i j k}\right)= \begin{cases}1 & \text { if } u_{i j k}=0 \\ 0 & \text { otherwise }\end{cases}
$$

Each unit impulse response function, $a_{i j k}\left(u_{i j k}\right)$ has a magnitude of one, when $u_{i j k}=0$. The distance, $d_{i j k}$ and number of reflections will affect the magnitude of echoes. The number of reflections in a path is equal to the order of image source and used to calculate path length. If the reflection coefficient of all wall are the same, the wall reflection coefficient, $r_{w}$ will be derived from absorption coefficient. $\mathrm{n}$ represents the total number of reflection of sound signal.

$$
\begin{array}{r}
\mathrm{N}=|\mathrm{i}|+|\mathrm{j}|+|\mathrm{k}| \\
r_{i j k}=r_{w}^{|i|+|j|+|k|} \\
b_{i j k} \alpha \frac{1}{d_{i j k}}
\end{array}
$$

$$
\begin{gathered}
e_{i j k}=b_{i j k} r_{i j k} \\
h(t)=\sum_{i=-n}^{n} \sum_{j=-n}^{n} \sum_{k=-n}^{n} a_{i j k} e_{i j k}
\end{gathered}
$$

The RIR is obtained from summation of the total magnitude of each echo and unit impulse response function.

\section{ACOUSTIC PARAMETERS}

The RIR is measured with the help of ISM, but that data set could not describe any information related to behavior of room. Therefor there is a need for acoustic parameters, to study the acoustic behavior of a room.

\section{A. Clarity}

This parameter expresses a balance between early and late arriving energy. It is ratio of the energy contained in the first $80 \mathrm{~ms}$ of sound, early reflection to the energy from $80 \mathrm{~ms}$ onwards until extinction means late reverberation. So that it is also known as early to late sound index. $C_{80}$ is more formally defined as:

$$
C_{80}=10 \log \frac{\int_{0}^{80} h^{2}(t) d t}{\int_{80}^{\infty} h^{2}(t) d t} d B
$$

$C_{80}$ is associated with the perception of music and there is a similar definition for the perception of speech, $C_{50}$, with a measure over the first $50 \mathrm{~ms}$. This parameter is used with $80 \mathrm{~ms}$ and $50 \mathrm{~ms}$ for speech and music respectively.

\section{B. Definition}

This parameter expresses a balance between early and not only the late energy, but the total energy of the signal. It is ratio of energy contain in early reflection to total energy of signal.

$$
D_{80}=\frac{\int_{0}^{80} h^{2}(t) d t}{\int_{0}^{\infty} h^{2}(t) d t} * 100 \%
$$

Both integrals must include the direct sound. Definition should be $100 \%$ that means the impulse response does not contain any components with delay in excess of $80 \mathrm{~ms}$ or $50 \mathrm{~ms}$.

\section{RESULTS}

The ISM is used to measure RIR of a rectangular room. The acoustic parameters are calculated from this measured RIR. The room is considered without door, window and furniture. The ceiling, floor and all walls of the room are assumed smoothly plastered. So the absorption coefficient is same for all the room surfaces at particular frequency. The source and receiver are assumed to be omni-directional.

The room size is $5 m \times 4 m \times 3 m$ and the position of receiver is $(2.5,2,1.5) \mathrm{m}$. These $(0.8,1,1,5) \mathrm{m},(1,0.2,0.9) \mathrm{m}$, $(3,0.5,0.5) \mathrm{m},(2.5,1.5,2) \mathrm{m}$ and $(2,1,2.5) \mathrm{m}$ five different positions are randomly chosen to place the source. The source is placed one after the other to measure RIR and after that clarity and definition will be calculated for all that position of source. The RIR is measured for six different frequencies such as $125 \mathrm{~Hz}, 250 \mathrm{~Hz}, 500 \mathrm{~Hz}, 1000 \mathrm{~Hz}, 2000 \mathrm{~Hz}$ and $4000 \mathrm{~Hz}$, the absorption coefficient is $0.013,0.015,0.02,0.03,0.04$ and 
0.05 respectively. The figure IV and $\mathrm{V}$ show that the mean clarity and definition of those five different positions which are calculated from the measured RIR.

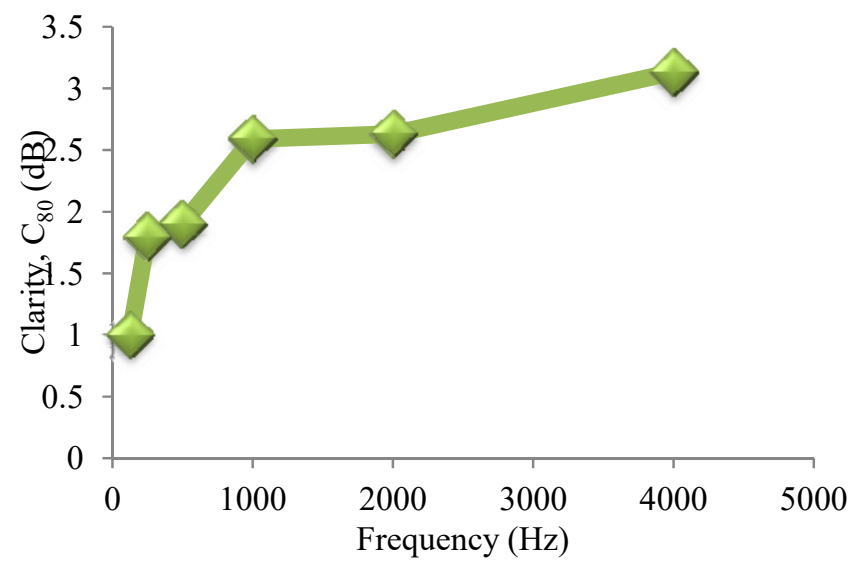

FIGURE IV. FREQUENCY VS. MEAN CLARITY: FOR FIVE DIFFERENT POSITION OF SOURCE

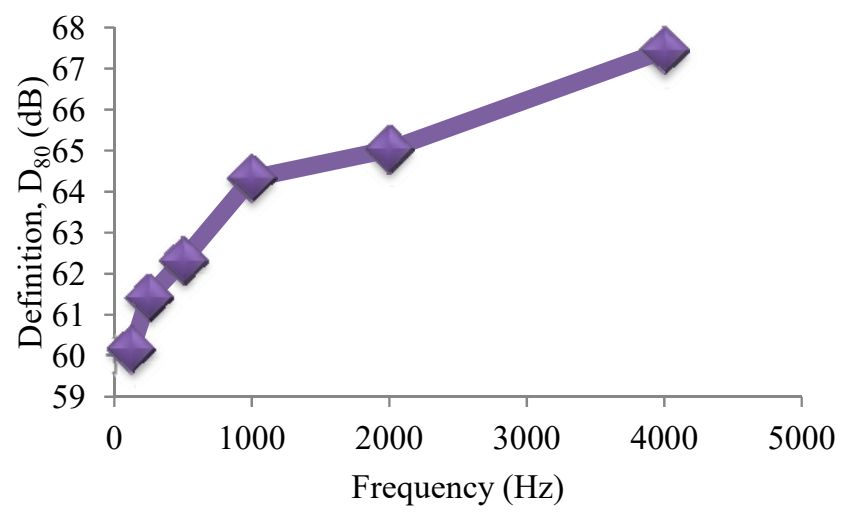

FIGURE V. FREQUENCY VS. DEFINITION: FOR FIVE DIFFERENT POSITION OF SOURCE

\section{CONCLUSION}

The RIR of a small rectangular room has measured from ISM which is computer simulation technique, if the source and receiver are assumed to be omni-directional and room surfaces are smoothly plastered. These measured RIR is used to calculate clarity and definition. The figure IV and V show that the mean clarity and definition increase as frequency or absorption coefficient increases. That means change in absorption coefficient or frequency can affect the RIR for all the position of source and receiver in the same manner.

\section{ACKNOWLEDGMENT}

The work was supported by the Department of Electronics and Communication of Dharmsinh Desai University, Nadiad.

\section{REFERENCES}

[1] F. Everest and K. Pohlman: Master Handbook of Acoustics. 5th Edition, McGraw-Hill, New York (2009).

[2] H. Kuttruff: Room acoustics, 5th Edition, Spon Press, New York (2009).
[3] J. Allen and D. Berkley: Image method for efficiently simulating smallroom acoustics. Journal of Acoustical Society of America, vol. 65. (1979) 943-950.

[4] J. Borish: Extension of the image model to arbitrary polyhedral. Journal of Acoustical Society of America, vol. 75. (1984) 1827-1836.

[5] J. Rindel: The use of computer modeling in room acoustics. Journal of Vibroengineering, vol. 3. (2000) 219-224.

[6] P. Fausti and A. Farina: Acoustic measurements in opera houses: Comparison between different techniques and equipment. Journal of Sound and Vibration, vol. 232 (2000)213-229.

[7] www.acousticalsurfaces.com/acoustic_IOI/101_13.htm Last access date 24 March, 2015. 